\title{
Indexed realizability for bounded-time programming with references and type fixpoints
}

\author{
Aloïs Brunel ${ }^{1}$ and Antoine Madet $^{2}$ \\ 1 Aloïs Brunel, Université Paris 13, Sorbonne Paris Cité, \\ LIPN, CNRS, UMR 7030, F-93430, Villetaneuse, France. \\ 2 Univ Paris Diderot, Sorbonne Paris Cité, \\ PPS, UMR 7126, CNRS, F-75205 Paris, France
}

\begin{abstract}
The field of implicit complexity has recently produced several bounded-complexity programming languages. This kind of language allows to implement exactly the functions belonging to a certain complexity class. We here present a realizability semantics for a higher-order functional language based on a fragment of linear logic called LAL which characterizes the complexity class PTIME. This language features recursive types and higher-order store. Our realizability is based on biorthogonality, step-indexing and is moreover quantitative. This last feature enables us not only to derive a semantical proof of termination, but also to give bounds on the number of computational steps needed by typed programs to terminate.
\end{abstract}

\section{Introduction}

Implicit computational complexity — This research field aims at providing machine-independent characterizations of complexity classes (such as polynomial time or logspace functions). One approach is to use type systems based on linear logic to control the complexity of higher-order functional programs. In particular, the so-called light logics (e.g. LLL [7, SLL [10]) have led to various type systems for the $\lambda$-calculus guaranteeing that a well-typed term has a bounded complexity [3. These logics introduce the modalities '!' (read bang) and '§' (read paragraph). By a fine control of the nesting of these modalities, which is called the depth, the duplication of data can be made explicit and the complexity of programs can be tamed. This framework has been recently extended to a higher-order process calculus [6] and a functional language with recursive definitions [19. Also, Amadio and Madet have proposed [15] a multi-threaded $\lambda$-calculus with higher-order store that enjoys an elementary time termination.

Quantitative realizability _ - Starting from Kleene, the concept of realizability has been introduced in different forms and has been shown very useful to build models of computational systems. In a series of works [1312, Dal Lago and Hofmann have shown how to extend Kleene realizability with quantitative informations in order to interpret subsystems of linear logic with restricted complexity. The idea behind Dal Lago and Hofmann's work is to consider bounded-time 
programs as realizers, where bounds are represented by elements of a resource monoid. In [5] the first author has shown how this quantitative extension fits well in a biorthogonality based framework (namely Krivine's classical realizability [9]) and how it relates to the notion of forcing.

Step-indexing _ - In order to give a semantical account of features like recursive or reference types, one has to face troublesome circularity issues. To solve this problem, Appel and McAllester [2] have proposed step-indexed models. The idea is to define the interpretation of a type as a predicate on terms indexed by numbers. Informally, a term $t$ belongs to the interpretation of a type $\tau$ with the index $k \in \mathbb{N}$ if when $t$ is executed for $k$ steps, it satisfies the predicate associated to $\tau$. Then, it is possible to define by induction on the index $k$ the interpretation of recursive or reference types. Step-indexing has been related to Gödel-Löb logic and the later operator $\triangleright$ [17].

Contributions - In this paper, we present a typed $\lambda$-calculus called $\lambda_{\mathrm{LAL}}^{\mathrm{Reg}, \mu}$ whose functional core is based on the light logic LAL [3]. We extend it with recursive types and higher-order store. Even in presence of these features, every program typable in $\lambda_{\mathrm{LAL}}^{\mathrm{Reg}, \mu}$ terminates in polynomial time. To prove termination in bounded-time, we propose a new quantitative realizability semantics with the following features:

- It is biorthogonality based, which permits a simple presentation and allows the possibility to interpret control operators (though it is only discussed informally in the conclusion of this paper).

- It is indexed, which permits to interpret higher-order store and recursive types. The particularity is that our model is indexed by depths (the nesting of modalities) instead of computational steps (like in step-indexing).

To our knowledge, this is the first semantics presenting at the same time quantitative, indexed and biorthogonality features.

Outline - Section 2 introduces the language $\lambda_{\mathrm{LAL}}^{\mathrm{Reg}, \mu}$ and its type system. In Section 3, we introduce the indexed quantitative realizability. It is then used to obtain a semantic model for $\lambda_{\mathrm{LAL}}^{\mathrm{Reg}, \mu}$, which in turn implies termination in polynomial time of typed programs. Finally, we mention related works in Section 4 and in Section 5 we discuss future research directions and conclude.

\section{The language}

This section presents the language $\lambda_{\mathrm{LAL}}^{\mathrm{Reg}, \mu}$ and its type system. Before going into details, we give some intuitions on the modalities bang and paragraph and explain how we deal with side-effects with the notion of region.

On bang and paragraph - The functional core of the language is an affine $\lambda$-calculus which means that functions use their argument at most once. 
Modal constructors '!' and ' $§$ ' originating from $\mathbf{L A L}$ are added to the language. Intuitively, from a value $V$ which can be used at most once, we can construct a value $! V$ which can be duplicated with a special modal binder. Values which have been duplicated are of the shape $\S V$ and therefore cannot be duplicated anymore. We will see more precisely with the type system how we can be polynomial with these modal operators.

On regions - Following a standard practice in effect systems [14, the global store is abstracted into a finite set of regions where each region represents one or several dynamic memory locations. Then, side-effects are produced by read and write operators on constant region names. As noted by Amadio [1], the abstract language with regions simulates the concrete language with dynamic memory locations as long as the values assigned to regions do not erase the previous ones. In particular, termination in polynomial time of the language with regions should entail termination in polynomial time of the language with references. There are two reasons for working with regions instead of memory locations. First, they allow to deal with several kinds of side-effects. Our language is sequential hence regions naturally represent higher-order references $\grave{a}$ la $\mathrm{ML}$, but in the context of concurrent programming they could represent communication channels or even signals in the context of reactive programming. Second, we find it easier to give a semantic model of a type system with regions instead of dynamic addresses.

\subsection{Syntax and operational semantics}

The syntax of the language is the following:

$$
\begin{array}{ll}
\text { Values } \quad V::= & x|\lambda x . M| r|()| \bar{n}|! V| \S V \\
\text { Terms } \quad M::= & V\left|M_{1} M_{2}\right| V_{1} \star V_{2}|! M| \S M \\
& \text { let } ! x=V \text { in } M \mid \text { let } \S x=V \text { in } M \\
& \operatorname{get}(r) \mid \operatorname{set}(r, V)
\end{array}
$$

We suppose having a countable set of variables denoted $x, y, \ldots$ and of regions denoted by the letters $r, r^{\prime}, \ldots$. The terminal value unit is denoted by (). Integers are denoted by $\bar{n}$ and $V_{1} \star V_{2}$ stands for any arithmetical operation. Modal terms and modal values are built with the unary constructors! and $\S$ and are destructed by the respective let $!$ and let $\S$ binders. The terms get $(r)$ and $\operatorname{set}(r, V)$ are respectively used to read a value from a region $r$ and to assign a value $V$ to a region $r$. As usual we write the sequential composition $M ; N$ for $(\lambda x . N) M$ where $x$ does not occur free in $N$. We denote by $M[N / x]$ the term $M$ in which each free occurrence of $x$ has been substituted by $N$.

The operational semantics of the language is presented in the form of an abstract machine. We first define the configurations of the abstract machine:

$$
\begin{array}{ll}
\text { Environments } & E::=\diamond|V \cdot E| M \odot E|! \cdot E| \S \cdot E \\
\text { Stores } & S::=r \Leftarrow V \mid S_{1} \uplus S_{2} \\
\text { Configurations } & C::=\langle M, E, S\rangle
\end{array}
$$


Programs are intended to be executed with a right-to-left call-by-value strategy. Hence, an environment $E$ is either an empty frame $\diamond$, a stack of frames to evaluate on the left of a value $(V \cdot E)$, on the right of a term $(M \odot E)$ or in-depth of a term $(! \cdot E$ and $\S \cdot E)$. Finally, a store $S$ is a multiset of region assignments $r \Leftarrow V$. A configuration of the abstract machine is executed according to the following rules:

$$
\begin{aligned}
\left\langle\overline{n_{1}} \star \overline{n_{2}}, E, S\right\rangle & \rightarrow\left\langle\overline{n_{1} \star n_{2}}, E, S\right\rangle \\
\langle\lambda x \cdot M, V \cdot E, S\rangle & \rightarrow\langle M[V / x], E, S\rangle \\
\langle M N, E, S\rangle & \rightarrow\langle N, M \odot E, S\rangle \\
\langle V, M \odot E, S\rangle & \rightarrow\langle M, V \cdot E, S\rangle \\
\langle\dagger M, E, S\rangle & \rightarrow\langle M, \dagger \cdot E, S\rangle \quad \text { if } M \text { is not a value } \\
\langle V, \dagger \cdot E, S\rangle & \rightarrow\langle\dagger V, E, S\rangle \\
\langle\text { let } \dagger x=\dagger V \text { in } M, E, S\rangle & \rightarrow\langle M[V / x], E, S\rangle \\
\langle\operatorname{get}(r), E, r \Leftarrow V \uplus S\rangle & \rightarrow\langle V, E, S\rangle \\
\langle\operatorname{set}(r, V), E, S\rangle & \rightarrow\langle(), E, r \Leftarrow V \uplus S\rangle
\end{aligned}
$$

For the sake of conciseness we wrote $\dagger$ for $\dagger \in\{!, \S\}$. Observe that the let $\dagger$-binders destruct modal values $\dagger V$ and propagate $V$. Therefore, a value $!^{n} V$ should be duplicable at least $n$ times. Reading a region amounts to consume the value from the store and writing to a region amounts to add the value to the store. We consider programs up to $\alpha$-renaming and in the sequel $\stackrel{*}{\rightarrow}$ denotes the reflexive and transitive closure of $\rightarrow$.

Example 1. Here is a function $F=\lambda x$.let $! y=x$ in $\operatorname{set}\left(r_{1}, \S y\right)$; set $\left(r_{2}, \S y\right)$ that duplicates its argument and assign it to regions $r_{1}$ and $r_{2}$. It can be used to duplicate a value from another region $r_{3}$ as follows:

$$
\left\langle F \operatorname{get}\left(r_{3}\right), \diamond, r_{3} \Leftarrow ! V\right\rangle \stackrel{*}{\rightarrow}\left\langle(), \diamond, r_{1} \Leftarrow \S V \uplus r_{2} \Leftarrow \S V\right\rangle
$$

Therefore the value $\S V$ stored in $r_{1}$ and $r_{2}$ is no longer duplicable.

Definition 1. We define the notation $\langle M, E, S\rangle \Downarrow^{n}$ as the following statement:

- The evaluation of $\langle M, E, S\rangle$ in the abstract machine terminates.

- The number of steps needed by $\langle M, E, S\rangle$ to terminate is $n$.

\subsection{Type system}

The light logic LAL relies on a stratification principle which is at the basis of our type system. We first give an informal explanation of this principle.

On stratification - Each occurrence of a program can be given a depth which is the number of nested modal constructors that it appears under. Here is 
an example for the program $P$ where each occurrence is labeled with its depth:

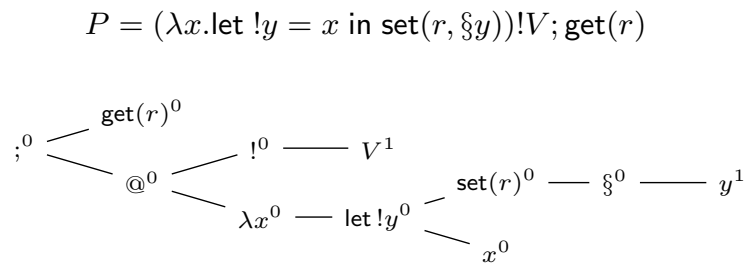

The depth $d(M)$ of a term $M$ is the maximum depth of its occurrences. The stratification principle is that the depth of every occurrence is preserved by reduction. On the functional side, it can be ensured by these two constraints: (1) if a $\lambda$-abstraction occurs at depth $d$, then it binds at depth $d ;(2)$ if a let $\dagger$ occurs at depth $d$, then it binds at depth $d+1$. These two constraints are respected by the program $P$ and we observe in the following reduction

$$
\langle P, \diamond, \emptyset\rangle \stackrel{*}{\rightarrow}\langle\operatorname{set}(r, \S V), \diamond, \emptyset\rangle
$$

that the depth of $V$ is preserved. In order to preserve the depth of occurrences that go through the store, this third constraint is needed: (3) for each region $r$, get $(r)$ and $\operatorname{set}(r)$ must occur at a fixed depth $d_{r}$. We observe that this is the case of program $P$ where $d_{r}=0$. Consequently, the reduction terminates as follows

$$
\langle\operatorname{set}(r, \S V), ! \cdot \diamond, \emptyset\rangle \stackrel{*}{\rightarrow}\langle\S V, \diamond, \emptyset\rangle
$$

where the depth of $V$ is still preserved. Stratification on the functional side has been deeply studied by Terui with the Light Affine $\lambda$-calculus [20] and extended to regions by Amadio and the second author [15].

We now present the type system for $\lambda_{\mathrm{LAL}}^{\mathrm{Reg}, \mu}$ that formalizes stratification and should ensure polynomial soundness.

Types and contexts _ - The syntax of types and contexts is the following:

$$
\begin{array}{lrl}
\text { Types } & A, B::=\alpha \mid \text { Unit }|\mathrm{N}| A \multimap B|! A| \S A|\mu X . A| \operatorname{Reg}_{r} A \\
\text { Variable contexts } & \Gamma, \Delta::=x_{1}:\left(u_{1}, A_{1}\right), \ldots, x_{n}:\left(u_{n}, A_{n}\right) \\
\text { Region contexts } & R::=r_{1}:\left(\delta_{1}, A_{1}\right), \ldots, r_{n}:\left(\delta_{n}, A_{n}\right)
\end{array}
$$

We have a countable set of type variables $X, X^{\prime}, \ldots$ Then, we distinguish the terminal type Unit, the type of integers $\mathrm{N}$, the affine functional type $A \multimap B$, the type ! $A$ of values that can be duplicated, the type $\S A$ of values that may have been duplicated, recursive types $\mu X . A$ and the type $\operatorname{Reg}_{r} A$ of regions $r$ containing values of type $A$. Hereby types may depend on regions. Following [15], a region context associates a natural number $\delta_{i}$ to each region $r_{i}$ of a finite set of regions $\left\{r_{1}, \ldots, r_{n}\right\}$ that we write $\operatorname{dom}(R)$. Writing $r:(\delta, A)$ means that the region $r$ contains values of type $A$ and that gets and sets on $r$ may only happen at a fixed depth depending on $\delta$. A variable context associates each variable 
with an usage $u \in\{\lambda, \S, !\}$ which constraints the variable to be bound by a $\lambda$ abstraction, a let $\S$-binder or a let !-binder respectively. In the sequel we write $\Gamma_{u}$ for $x_{1}:\left(u, A_{1}\right), \ldots, x_{n}:\left(u, A_{n}\right)$. Writing $x:(u, A)$ means that the variable $x$ ranges on values of type $A$ and can be bound according to $u$.

Types depend on region names. As we shall see, this allows for a straightforward interpretation of the type $\operatorname{Reg}_{r} A$. Moreover, it induces a typed translation from a language with dynamic locations to a language with regions. For example, for every occurrence $P$ of dynamic allocation like let $x=\operatorname{ref} M$ in $N$ in an ML program where $M$ is of type $A$, it suffices to introduce a distinct region name $r$ and associate the variable $x$ with type $\operatorname{Reg}_{r} A$. Then, the type preserving translation of $P$ is simply $\operatorname{set}(r, M) ; N[r / x]$. However, we have to be careful in stating when a type $A$ is well-formed with respect to a region context $R$, written $R \vdash A$. Informally, the judgment $r_{1}:\left(\delta_{1}, A_{1}\right), \ldots, r_{n}:\left(\delta_{n}, A_{n}\right) \vdash B$ is well formed provided that: (1) all the region names occurring in the types $A_{1}, \ldots, A_{n}, B$ belong to the set $\left\{r_{1}, \ldots, r_{n}\right\},(2)$ all types of the shape $\operatorname{Reg}_{r_{i}} B$ with $i \in\{1, \ldots, n\}$ and occurring in the types $A_{1}, \ldots, A_{n}, B$ are such that $B=A_{i}$. The judgment $R \vdash \Gamma$ is well-formed if $R \vdash A$ is well-formed for every $x:(u, A) \in \Gamma$. We invite the reader to check in [1] that these judgements can be easily defined.

Typing rules - A typing judgment takes the form $R ; \Gamma \vdash^{\delta} P: A$ and is indexed by an integer $\delta$. The rules are given in Figure 1 They should entail the following:

- if $x:(\lambda, A) \in \Gamma$ then $x$ occurs at most once at depth 0 in $P$,

- if $x:(\S, A) \in \Gamma$ then $x$ occurs at most once at depth 1 in a subterm $\S M$ of $P$,

- if $x:(!, A) \in \Gamma$ then $x$ occurs arbitrarily many times at depth 1 in a subterm $\S M$ or $! M$ of $P$,

- if $r:\left(\delta^{\prime}, A\right) \in R$ then get $(r)$ and $\operatorname{set}(r)$ occur at depth $\delta-\delta^{\prime}$ in $P$.

Several remarks have to be made:

- In binary rules, we implicitly require that contexts $\Gamma$ and $\Delta$ are disjoints. They are explicit rules for the weakening and contraction of variables and we may only contract variables with usage !. Therefore, let!s are the only operators that can bind several occurrences of a variable.

- There are two restrictions to apply the rule !-prom: first, $V$ may contain at most one occurrence of a free variable; second, $V$ is a value (we cannot type $! M)$. The first constraint ensures that the size of a program does not explode exponentially and is well studied in [20. The second condition is due to sideeffects. To see this, assume that we can type $! M$. Then we can derive the judgment $r:(0, A) ;-\vdash^{1} \lambda x$.let ! $y=x$ in $\S \operatorname{set}(r, x) ; ! \operatorname{get}(r): \S A \multimap ! A$. Both get $(r)$ and $\operatorname{set}(r)$ occur at depth 1 but under different modalities. Clearly the type $\S A \multimap ! A$ which cannot be derived in LAL has to be rejected for otherwise we can freely re-duplicate a value $\S V$. 


$$
\begin{aligned}
& \text { int } \frac{R \vdash}{R ;-\vdash^{\delta} \bar{n}: \mathrm{N}} \quad \operatorname{arith} \frac{R ; \Gamma \vdash^{\delta} V_{1}: \mathrm{N} \quad R ; \Delta \vdash^{\delta} V_{2}: \mathrm{N}}{R ; \Gamma, \Delta \vdash^{\delta} V_{1} \star V_{2}: \mathrm{N}} \\
& \mathrm{v} \frac{R \vdash}{R ; x:(\lambda, A) \vdash^{\delta} x: A} \quad \mathrm{u} \frac{R \vdash}{R ;-\vdash^{\delta}(): \text { Unit }} \quad \mathrm{r} \frac{R \vdash \quad r:(\delta, A) \in R}{R ;-\vdash^{\delta} r: \operatorname{Reg}_{r} A} \\
& \mathrm{c} \frac{R ; \Gamma, x:(!, A), y:(!, A) \vdash^{\delta} M: B}{R ; \Gamma, z:(!, A) \vdash^{\delta} M[z / x, z / y]: B} \mathrm{w} \frac{R ; \Gamma \vdash^{\delta} M: B \quad R \vdash \Gamma, x:(u, A)}{R ; \Gamma, x:(u, A) \vdash^{\delta} M: B} \\
& \operatorname{lam} \frac{R ; \Gamma, x:(\lambda, A) \vdash^{\delta} M: B}{R: \Gamma \vdash^{\delta} \lambda x \cdot M: A \multimap B} \text { app } \frac{R ; \Gamma \vdash^{\delta} M_{1}: A \multimap B \quad R ; \Delta \vdash^{\delta} M_{2}: A}{R ; \Gamma, \Delta \vdash^{\delta} M_{1} M_{2}: B} \\
& \text { !-prom } \frac{R ; x:(\lambda, A) \vdash^{\delta} V: A}{R ; x:(!, A) \vdash^{\delta+1} ! V: ! A} \S \text {-prom } \frac{R ; \Gamma_{\lambda}, \Delta_{\lambda} \vdash^{\delta} M: A}{R ; \Gamma_{\S}, \Delta_{!} \vdash^{\delta+1} \S M: \S A} \\
& R ; \Gamma \vdash^{\delta} V: \dagger A \\
& \dagger \text {-elim } \frac{R ; \Delta, x:(\dagger, A) \vdash^{\delta} M: B}{R ; \Gamma, \Delta \vdash^{\delta} \text { let } \dagger x=V \text { in } M: B} \operatorname{get} \frac{R ;-\vdash^{\delta} r: \operatorname{Reg}_{r} A}{R ;-\vdash^{\delta} \operatorname{get}(r): A} \\
& R ;-\vdash^{\delta} r: \operatorname{Reg}_{r} A \\
& \text { set } \frac{R ; \Gamma \vdash^{\delta} V: A}{R ; \Gamma \vdash^{\delta} \operatorname{set}(r, V): \text { Unit }} \text { un } / \text { fold } \frac{R ; \Gamma \vdash^{\delta} M: \mu X . A}{R ; \Gamma \vdash^{\delta} M: A[\mu X . A / X]}
\end{aligned}
$$

Fig. 1. Typing rules

- The depth $\delta$ of a judgment is incremented when we construct a modal term. This allows to count the number of nested modalities and to stratify regions by requiring that the depth of a region matches the depth of the judgment in the rule $\mathrm{R}$.

- For space consideration the rule un/fold can be used upside down.

Definition 2. We say that a program $M$ is well-typed if a judgment $R ; \Gamma \vdash^{\delta}$ $M: A$ can be derived for some $R, \Gamma$ and $\delta$ such that:

- If $r:\left(\delta_{r}, A\right) \in R$ then $A=\S B$.

- For every type fixpoint $\mu X$.A that appears in $R$ and $\Gamma$, the occurrences of $X$ in $A$ are guarded by (occur under) a modality $\dagger$.

- Every depth index in the derivation is positive. Note that if this is not the case, we can always find $\delta^{\prime}>\delta$ such that this is true for $R ; \Gamma \vdash^{\delta^{\prime}} M: A$.

These three conditions will be needed to give a well-founded interpretation.

Example 2. The operational semantics of references (values are copied from the store) can be simulated as long as values stored in regions are of the shape $! V$. For example, consider the following well-typed program

$$
r:(0, \mathrm{~N}) ;-\vdash^{1} \text { let } ! x=\operatorname{get}(r) \text { in } \operatorname{set}(r, ! x) ; \S(x * x): \mathrm{N}
$$


that reduces as follows:

$$
\langle\text { let } ! x=\operatorname{get}(r) \text { in } \operatorname{set}(r, ! x) ; \S(x * x), \diamond, r \Leftarrow ! \bar{n}\rangle \stackrel{*}{\rightarrow}\langle\S(\overline{n * n}), \diamond, r \Leftarrow ! \bar{n}\rangle
$$

Indeed, the region $r$ can be considered a reference since the value $! \bar{n}$ has not been erased from the store.

The following progress property can be derived as long the program do not try to read an empty region.

Proposition 1 (Progress). If $R ; \Gamma \vdash^{\delta} M: A$ then $\langle C, \diamond, \emptyset\rangle \stackrel{*}{\rightarrow}\langle V, \diamond, S\rangle$ and $R ; \Gamma \vdash^{\delta} V: A$ and every assigned value in $S$ can be typed.

The goal of the next section is to prove the following theorem

Theorem 1 (Polynomial termination). There exists a family of polynomials $\left\{P_{d}\right\}_{d \in \mathbb{N}}$ such that if $M$ is well-typed then $\langle M, \diamond, \diamond\rangle$ terminates in at most $P_{d(M)}(\operatorname{size}(M))$ steps.

\section{3 "Indexed" quantitative realizability}

We now present a biorthogonality-based interpretation of $\lambda_{\mathrm{LAL}}^{\mathrm{Reg}, \mu}$. Apart from the use of biorthogonality, this interpretation has two particularities:

- First, the realizability model is quantitative. A type is interpreted by a set of weighted realizers (that is a program together with a store and a quantity bounding its normalization time). This allows to prove complexity properties of programs.

- Secondly, the semantics is indexed (or stratified), meaning that we interpret a type by a family of sets indexed by $\mathbb{N}$. Moreover the interpretation of a type is defined by double induction, first on the index $n$, and secondly on the size of the type. This allows to interpret recursive types and references.

It is worth noticing that while our interpretation is similar to the so-called "stepindexed" models, the meaning of indexes is not (directly) related to the number of computation steps but to the depth of terms (and so our model could be described as a "depth-indexed" model). It is the quantitative part which is used to keep track of the number of computational steps.

\subsection{The light monoid}

The realizability model is parametrized by a monoid, whose elements represent an information about the amount of time needed by a program to terminate. To interpret $\lambda_{\mathrm{LAL}}^{\mathrm{Reg}, \mu}$, we use the light monoid, which is a simplification of a resource monoid introduced in 12 .

Definition 3. The light monoid is defined as the structure $(\mathcal{M},+,\|\cdot\|, !, \S)$ where 
- $\mathcal{M}$ is the set of triples $(n, m, f)$ such that $n, m \in \mathbb{N}$ and $f: \mathbb{N} \rightarrow \mathbb{N}$ is increasing.

- If $(n, m, f),(l, k, g) \in \mathcal{M},(n, m, f)+(l, k, g)=(n+l, \max (m, k), \max (f, g))$.

- If $(n, m, f) \in \mathcal{M},\|(n, m, f)\|=n f(m+n)$.

- If $(n, m, f) \in \mathcal{M}, \S(n, m, f)=\left(n / m, m, x \mapsto x^{2} f\left(x^{2}\right)\right)$.

- Finally, for any $(n, m, f) \in \mathcal{M}, !(n, m, f)=\left(1, n+m, x \mapsto x^{3} f\left(x^{3}\right)\right)$.

We moreover denote by $\boldsymbol{n}$ the element of $\mathcal{M}$ defined as $(n, 0, x \mapsto 0)$.

From now on, we use lower-case consonnes letters $p, q, m, v, \ldots$ to denote elements of $\mathcal{M}$. To give some intuitions on these operations, let's say that + represents the resource consumption resulting of the interaction of two programs, and that $\|p\|$ calculates the concrete bound (a natural number) associated to an abstract bound $p \in \mathcal{M}$.

Remark 1. The structure $(\mathcal{M},+, \mathbf{0},\|\cdot\|)$ is a quantitative monoid in the sense of [5]. In particular, it satisfies the following inequality:

$$
\forall p, q,\|p\|+\|q\| \leq\|p+q\|
$$

This inequality informally represents the fact that the amount of resource consumed by the interaction of two programs is more than the total amount of resource used by the two programs alone.

Definition 4. Given $p, q \in \mathcal{M}$, we say that $p<q$ iff $\forall r \in \mathcal{M},\|p+r\|<\|q+r\|$ and $p \leq q$ iff $\forall r \in \mathcal{M},\|p+r\| \leq\|q+r\|$

Property 1. The relation $\leq$ enjoy the following properties:

- If $p \leq q$, then $p+r \leq q+r$.

- If $p \leq q$, then $\|p\| \leq\|q\|$.

- If $p \leq p^{\prime}$ and $q \leq q^{\prime}$, then $p+q \leq q+q^{\prime}$.

Property 2. The operations! and $\S$ on the monoid $\mathcal{M}$ satisfy the following properties:

$-\S p \leq ! p$

$-\S(p+q) \leq \S p+\S q$

$-\S p+\S q \leq \S(p+q)+\mathbf{2}$

$-! p+! p=! p+1$

A third operation $F$ will be used to interpret functoriality of !:

Property 3. Let $p=(n, m, f)$ and $q$ two elements of $\mathcal{M}$. We define $F(p)$ to be $\left(1+n+m, m, x \mapsto x^{3} f\left(x^{3}\right)\right)$. We then have $!(p+q) \leq F(p)+! q$.

Finally we define a notion of $\mathcal{M}$-context, which is similar to an evaluation context, but for resource bounds instead of programs.

Definition 5. A $\mathcal{M}$-context is any function $f: \mathcal{M} \rightarrow \mathcal{M}$ obtained by composition of the functions $\lambda x . x+p$ (where $p$ is any element of $\mathcal{M}$ ), $\lambda x . ! x$ and $\lambda x . \S x$. The set of $\mathcal{M}$-contexts is denoted by $\mathcal{M}[$.$] .$ 
Example 3. For instance, $\lambda x .(!(! x+\mathbf{1}))$ is a valid $\mathcal{M}$-context, but $\lambda x .(! x+x+\S x)$ and $\lambda x .0$ are not.

Any $\mathcal{M}$-context $f$ is monotonic in its argument, that is if $p<q$, then $f(p)<f(q)$ and if $p \leq q$, then $f(p) \leq f(q)$.

\subsection{Orthogonality}

Usually, orthogonality is defined between a program and an environment. Here, it defined between a weighted program and a weighted environment.

Definition 6. - A weighted term is a tuple $(M, p)$ where $M$ is a closed term and $p$ an element of $\mathcal{M}$. The set of weighted terms is denoted by $\Lambda_{\mathcal{M}}$.

- A weighted stack is a pair $(E, e)$ where $E$ is a closed stack and e an element of $\mathcal{M}[$.$] . The set of weighted stacks is denoted by \Pi_{\mathcal{M}}$.

We choose a pole $\Perp \subseteq$ Conf $\times \mathcal{M}$ as the set of bounded-time terminating weighted configurations:

$$
\Perp=\left\{(\langle M, E, S\rangle, p) \mid\langle M, E, S\rangle \Downarrow^{n} \wedge n \leq\|p\|\right\}
$$

In orthogonality-based models, fixing a pole, also called observable, corresponds to choose a notion of correct computation.

Proposition 2. This pole satisfies some important properties:

1. ( $\leq$-saturation) If $(\langle M, E, S\rangle, p) \in \Perp$ and $p \leq q$ then $(\langle M, E, S\rangle, q) \in \Perp$.

2. ( $\rightarrow$-saturation) If $(\langle M, E, S\rangle, p) \in \Perp$ and $\left\langle M^{\prime}, E, S^{\prime}\right\rangle \rightarrow\langle M, E, S\rangle$ then $\left(\left\langle M^{\prime}, E^{\prime}, S^{\prime}\right\rangle, p+1\right) \in \Perp$.

The pole induces a notion of orthogonality. In contrast with usual models, since we need to deal with references, the orthogonality relation is parametrized by a set $\mathcal{S}$ of stores.

Definition 7. The orthogonality relation $\perp_{\mathcal{S}} \subseteq \Lambda_{\mathcal{M}} \times \Pi_{\mathcal{M}}$ is defined as:

$$
(M, p) \perp_{\mathcal{S}}(E, e) \quad \text { iff } \forall(S, s) \in \mathcal{S},(\langle M, E, S\rangle, e(p+s)) \in \Perp
$$

This orthogonality relation lifts to sets of weighted terms and weighted stacks. If $X \subseteq \Lambda_{\mathcal{M}}\left(\operatorname{resp} X \subseteq \Pi_{\mathcal{M}}\right)$,

$$
\begin{gathered}
X^{\perp_{\mathcal{S}}}=\left\{(E, e) \in \Pi_{\mathcal{M}} \mid \forall(M, p) \in X,(M, p) \perp_{\mathcal{S}}(E, e)\right\} \\
\left(\text { resp. } X^{\perp_{\mathcal{S}}}=\left\{(M, p) \in \Lambda_{\mathcal{M}} \mid \forall(E, e) \in X,(M, p) \perp_{\mathcal{S}}(E, e)\right\}\right)
\end{gathered}
$$

The operation $(.)^{\perp_{\mathcal{S}}}$ satisfies the usual orthogonality properties.

Lemma 1. Suppose $X, Y \subseteq \Lambda_{\mathcal{M}}$ or $X, Y \subseteq \Pi_{\mathcal{M}}$ :

1. $X \subseteq Y$ implies $Y^{\perp \mathcal{S}} \subseteq X^{\perp \mathcal{S}}$ 
2. $X \subseteq X^{\perp_{S} \perp_{S}}$

3. $X^{\perp_{\mathcal{S}} \perp_{\mathcal{S}} \perp_{\mathcal{S}}}=X^{\perp_{\mathcal{S}}}$

Definition 8. If $X$ is a set of weighted realizers, we define its $\leq$-closure $\bar{X}=$ $\{(M, p) \mid \exists q \leq p,(M, q) \in X\}$.

Remark 2. Notice that for any $\mathcal{S}$, we have $\bar{X} \subseteq X^{\perp_{\mathcal{S}} \perp_{\mathcal{S}}}$.

We say that a set $X \subseteq \Lambda_{\mathcal{M}}$ is a $\mathcal{S}$-behavior if $X=X^{\perp_{\mathcal{S}} \perp_{\mathcal{S}}}$. Finally, we can define the set of $\mathcal{S}$-reducibility candidates. To do that, we first need to extend the language of terms with a new constant

$$
M \quad::=\mid
$$

This constant comes with no particular reduction rule. It can be seen as a special variable considered as a closed term and is in a sense the dual of the empty stack.

Definition 9. The set of $\mathcal{S}$-reducibility candidates, denoted by $\mathrm{CR}_{\mathcal{S}}$ is the set of $\mathcal{S}$-behaviors $X$ such that $(\boldsymbol{\Psi}, \boldsymbol{0}) \in X \subseteq\{(\diamond, x \mapsto x)\}^{\perp_{\mathcal{S}}}$

Remark 3. If $(M, p) \in X$ where $X$ is a $\mathcal{S}$-reducibility candidate and if $(\diamond, \mathbf{0}) \in \mathcal{S}$, then $\langle M, \diamond, \diamond\rangle$ terminates in at most $\|p\|$ steps. In fact our notion of reducibility candidate extends the usual notion in the non-quantitative case.

Finally, suppose $R$ is a set of regions and suppose $\mathcal{S}_{R}$ is a set of stores whose domain is restricted to a $R$. We say that:

$\mathcal{S}_{R} \sqsubseteq \mathcal{S}^{\prime} \Leftrightarrow \mathcal{S}^{\prime}$ contains $\mathcal{S}_{R}$ and if $(S, s) \in \mathcal{S}^{\prime}$ and if we write $S=S^{\delta} \uplus S^{\prime \prime}$, then there is a decomposition $s=s^{\prime}+s^{\prime \prime}$ such that $\left(S^{\delta}, s^{\prime}\right) \in \mathcal{S}_{R}$ and moreover, if $\left(S_{R}, s_{R}\right) \in \mathcal{S}_{R}$ then $\left(S^{\prime \prime} \uplus S_{R}, s^{\prime \prime}+s_{R}\right) \in \mathcal{S}^{\prime}$.

Remark 4. This quite involved definition will permit to the interpretation of a type to enjoy properties similar to the one called extension/restriction in [1]. In other words, given a store, it gives a way to say what substore can be removed safely and what stores can be added to it safely.

\subsection{Interpretation of $\lambda_{\mathrm{LAL}}^{\mathrm{Reg}, \mu}$}

Using the orthogonality machinery previously defined, we can give an interpretation of $\lambda_{\mathrm{LAL}}^{\mathrm{Reg}, \mu}$ types as reducibility candidates. Suppose $R$ is the following region context:

$$
R=r_{1}:\left(\delta_{1}, \S A_{1}\right), \ldots, r_{n}:\left(\delta_{n}, \S A_{n}\right)
$$

We define three indexed sets: the interpretation $|R|_{\delta}$ of the region context $R$, the pre-interpretation $\|R \vdash A\|_{\delta}$ of a type $A$ and its interpretation $|R \vdash A|_{\delta}^{\mathcal{S}}$ with 
respect to a set of stores $\mathcal{S}$. These three notions are defined by mutual induction, first on the index $\delta$, and then on the size of the type $A$.

$$
\begin{aligned}
|R|_{=\delta}= & \left\{\left(S, \sum_{\delta_{i}=\delta} \sum_{1 \leq j \leq k_{i}} \S q_{j}^{i}\right) \mid \operatorname{dom}(S)=\left\{r_{i} \mid r_{i}:\left(\delta_{i}, \S A_{i}\right) \in R \wedge \delta_{i}=\delta\right\}\right. \\
& \wedge \forall r_{i} \in \operatorname{dom}(S), S\left(r_{i}\right)=\left\{\S V_{1}^{i}, \S V_{2}^{i}, \ldots, \S V_{k_{i}}^{i}\right\} \\
& \left.\wedge \forall j \in\left[1, k_{i}\right],\left(V_{j}^{i}, q_{j}^{i}\right) \in \mathbb{\| R \vdash A _ { i } \| _ { \delta _ { i } - 1 }}\right\} \\
|R|_{\delta+1}= & \left\{\left.(S, s)\left|\exists\left(S_{1}, s_{1}\right) \in\right| R\right|_{=\delta+1}, \exists\left(S_{\delta}, s_{\delta}\right) \in|R|_{\delta}, S=S_{1} \uplus S_{\delta} \wedge s=s_{1}+\S s_{\delta}\right\}
\end{aligned}
$$

For convenience, we start the indexing of the interpretation at -1 instead of 0 .

$$
\|R \vdash A\|_{-1}=\{(\mathbf{H}, \mathbf{0})\}
$$

For $\delta \geq 0$, we define the pre-interpretation as:

$$
\begin{aligned}
\|R \vdash \mathrm{N}\|_{\delta} & =\{(\bar{n}, \mathbf{0}) \mid n \in \mathbb{N}\} \\
\left\|R \vdash U_{n i t}\right\|_{\delta} & =\{((), \mathbf{0})\} \\
\left\|R \vdash \operatorname{Reg}_{r} A\right\|_{\delta} & =\{(r, \mathbf{0})\} \\
\|R \vdash A \multimap B\|_{\delta} & =\left\{\left.(\lambda x . M, p)\left|\forall(V, v) \in\|R \vdash A\|_{\delta}, \forall \mathcal{S},\right| R\right|_{\delta} \sqsubseteq \mathcal{S},(M[V / x], p+v) \in|R \vdash B|_{\delta}^{\mathcal{S}}\right\} \\
\|R \vdash \S A\|_{\delta} & =\left\{(\S V, \S v) \mid(V, v) \in\|R \vdash A\|_{\delta-1}\right\} \\
\|R \vdash ! A\|_{\delta} & =\left\{(! V, ! v) \mid(V, v) \in\|R \vdash A\|_{\delta-1}\right\} \\
\|R \vdash \mu X . A\|_{\delta} & =\|R \vdash A[\mu X . A / X]\|_{\delta}
\end{aligned}
$$

The interpretation of a type with respect to a set $\mathcal{S}$ is just defined as the biorthogonal of the pre-interpretation:

$$
|R \vdash A|_{\delta}^{\mathcal{S}}=\|R \vdash A\|_{\delta}^{\perp_{\mathcal{S}} \perp_{\mathcal{S}}}
$$

Remark 5. Because of the presence of type fixpoints and regions, there are several circularities that could appear in the definition of $\|R \vdash A\|_{\delta}$. Yet, the interpretation is well defined for the following reasons:

- The type fixpoints $\mu X$. $A$ we consider are such that every occurrence of $X$ in $A$ is guarded by a modality ! or $\S$. But these modalities make the index of the interpretation decrease by one. Hence, $\|R \vdash \mu X . A\|_{\delta+1}$ is well defined as soon as $\|R \vdash \mu X . A\|_{\delta}$ is.

- To define $\|R \vdash A\|_{\delta+1}$, we need $|R|_{\delta+1}$ to be already defined. But here again, in $R$ each type is guarded by a modality $\S$. This implies that to define $|R|_{\delta+1}$, we only need to know each $\left\|R \vdash A_{i}\right\|_{\delta}$.

An important point is that the interpretation of a formula $A$ with respect to a region context $R$ and to an index $\delta \in \mathbb{N}$ is a $|R|_{\delta}$-reducibility candidate (it will be used to prove bounded-time termination).

Proposition 3. For all $\delta \in \mathbb{N}$ we have $|R \vdash A|_{\delta}^{|R|_{\delta}} \in \mathrm{CR}_{|R|_{\delta}}$.

This is to prove the inductive case of the arrow $\multimap$ that the use of the constant is mandatory. Indeed it is used in the proof to reduce under the $\lambda$ s. 


\subsection{Adequacy and bounded-time termination}

$$
\begin{aligned}
& \mathrm{v} \overline{\vdash^{\delta} x: \mathbf{0}} \quad \mathrm{r} \overline{\vdash^{\delta} r: \mathbf{0}} \quad \mathrm{u} \overline{\vdash^{\delta}(): \mathbf{0}}
\end{aligned}
$$

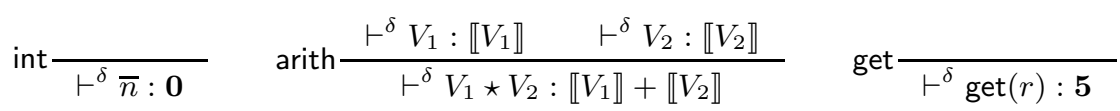

$$
\begin{aligned}
& \text { set } \frac{\vdash^{\delta} V: \llbracket V \rrbracket}{\vdash^{\delta+1} \operatorname{set}(r, \S V): \S \llbracket V \rrbracket+1} \quad \text { fold } \frac{\vdash^{\delta} M: \llbracket M \rrbracket}{\vdash^{\delta} M: \llbracket M \rrbracket} \quad \text { unfold } \frac{\vdash^{\delta} M: \llbracket M \rrbracket}{\vdash^{\delta} M: \llbracket M \rrbracket} \\
& \mathrm{c} \frac{x: !, y: ! \vdash^{\delta} M: \llbracket M \rrbracket}{z: ! \vdash^{\delta} M[z / x, z / y]: \llbracket M \rrbracket+1} \quad \mathrm{w} \frac{\vdash^{\delta} M: \llbracket M \rrbracket}{x: \delta \vdash^{\delta} M: \llbracket M \rrbracket} \\
& \operatorname{lam} \frac{\vdash^{\delta} M: \llbracket M \rrbracket}{\vdash^{\delta} \lambda x \cdot M: \llbracket M \rrbracket} \quad \text { app } \frac{\vdash^{\delta} M_{1}: \llbracket M_{1} \rrbracket \quad \vdash^{\delta} M_{2}: \llbracket M_{2} \rrbracket}{\vdash^{\delta} M_{1} M_{2}: \llbracket M_{1} \rrbracket+\llbracket M_{2} \rrbracket+\mathbf{3}} \\
& \S \text {-prom } \frac{\vdash^{\delta} M: \llbracket M \rrbracket}{\vdash^{\delta+1} \S M: \S \llbracket M \rrbracket+4} \quad \S \text {-elim } \frac{\vdash^{\delta} V: \llbracket V \rrbracket \quad \Gamma \vdash^{\delta} M: \llbracket M \rrbracket}{\vdash^{\delta} \text { let } \S x=V \text { in } M: \llbracket M \rrbracket+\llbracket V \rrbracket+\mathbf{3}} \\
& \text { !-prom } \frac{\vdash^{\delta} M: \llbracket M \rrbracket}{\vdash^{\delta+1} ! M: F(\llbracket M \rrbracket)} \quad \text { !-elim } \frac{\vdash^{\delta} V: \llbracket V \rrbracket \quad \Gamma \vdash^{\delta} M: \llbracket M \rrbracket}{\vdash^{\delta} \text { let } ! x=V \text { in } M: \llbracket M \rrbracket+\llbracket V \rrbracket+\mathbf{3}}
\end{aligned}
$$

Table 1. Inferring a bound from a $\lambda_{\mathrm{LAL}}^{\mathrm{Reg}, \mu}$ typing judgment

We now prove the soundness of our model with respect to $\lambda_{\mathrm{LAL}}^{\mathrm{Reg}, \mu}$ and as a corollary the bounded-time termination theorem.

In Table 1 is described how to infer an element of $\mathcal{M}$ from a $\lambda_{\mathrm{LAL}}^{\mathrm{Reg}, \mu}$ typing judgment: the notation $\llbracket M \rrbracket$ corresponds to the element of $\mathcal{M}$ already inferred from the typing judgment of $\llbracket M \rrbracket$, and each rule corresponds to the way $\llbracket M \rrbracket$ is built.

Definition 10. We use the notations $\bar{V}, \bar{p}$ and $\bar{y}$ to denote respectively a list of values $\left[V_{1}, \ldots, V_{n}\right]$, a list $\left[p_{1}, \ldots, p_{n}\right]$ of elements of $\mathcal{M}$ and a list of variables $\left[y_{1}, \ldots, y_{n}\right]$. If $M$ is a term, we denote by $M[\bar{V} / \bar{y}]$ the term $M\left[V_{1} / y_{1}, \ldots, V_{n} / y_{n}\right]$. If $\bar{p}$ is a list of elements of $\mathcal{M}$ and $\dagger \in\{!, \S\}$, we denote by $\dagger \bar{p}$ the list $\left[\dagger p_{1}, \ldots, \dagger p_{n}\right]$. We also define $\sum \bar{p}$ to be the sum $\sum_{1 \leq i \leq n} p_{i}$.

If $A$ is a type then we define $\lambda A$ as $A$ itself. Suppose $\Gamma=x_{1}:\left(e_{1}, A_{1}\right), \ldots, x_{n}$ : $\left(e_{n}, A_{n}\right)$. Then the notation $(\bar{V}, \bar{p}) \Vdash^{\delta} \Gamma$ stands for $\left(W_{i}, p_{i}\right) \in\left\|R \vdash e_{i} A_{i}\right\|_{\delta}$ for $1 \leq i \leq n$ with $W_{i}=V_{i}$ if $e_{i}=\lambda$ and $W_{i}=\dagger V_{i}$ if $e_{i}=\dagger$.

Example 4. If we have $(\bar{V}, \bar{p}) \Vdash^{\delta}\left(x_{1}:\left(\lambda, A_{1}\right), x_{2}:\left(\S, A_{2}\right), x_{3}:\left(!, A_{3}\right)\right)$ then $\bar{V}=$ $\left[V_{1}, V_{2}, M_{3}\right]$ and $\bar{p}=\left[p_{1}, \S p_{2}, ! p_{3}\right]$ such that $\left(V_{1}, p_{1}\right) \in\left\|R \vdash A_{1}\right\|_{\delta},\left(\S V_{2}, \S p_{2}\right) \in$ $\left\|R \vdash \S A_{2}\right\|_{\delta}$ and $\left(! V_{3}, ! p_{3}\right) \in\left\|R \vdash ! A_{3}\right\|_{\delta}$. 
Theorem 2 (Adequacy). Suppose that $R ; \Gamma \vdash^{\delta} M: C$. Let $(\bar{V}, \bar{p}) \Vdash^{\delta} \Gamma$, Then, for any $\mathcal{S}$ such that $|R|_{\delta} \sqsubseteq \mathcal{S}$,

$$
\left(M[\bar{V} / \bar{x}], \llbracket M \rrbracket+\sum \bar{p}\right) \in|R \vdash C|_{\delta}^{\mathcal{S}}
$$

Moreover, if $M$ is a value, then we have $\left(M[\bar{V} / \bar{x}], \llbracket M \rrbracket+\sum \bar{p}\right) \in \overline{\|R \vdash C\|_{\delta}}$.

Before giving the proof of Theorem 2, let's begin by an essential remark about the definition of the interpretation of a region context $R$.

Remark 6. Let $|R|_{\delta+1} \sqsubseteq R^{\prime}$. If $(S, s) \in R^{\prime}$, it can be uniquely written $\left(S^{\leq \delta} \uplus\right.$ $\left.S^{=\delta+1} \uplus S_{r}, \oint s_{\delta}+s_{\delta+1}+s_{r}\right)$ where $\left(S^{\leq \delta}, s_{\delta}\right) \in|R|_{\delta},\left(S^{\delta+1}, s_{\delta+1}\right) \in|R|_{=\delta+1}$ and $\operatorname{dom}\left(S_{r}\right) \subseteq\left\{r_{i} \mid \delta_{i}>\delta+1\right\}$. Hence, if we form the set $R_{\delta}^{\prime}=\{(S, s) \mid(S, \S s+$ $\left.\left.s_{\delta+1}+s_{r}\right) \in R^{\prime} \wedge\left(S^{\leq \delta}, s\right) \in|R|_{\delta}\right\}$ is such that $|R|_{\delta} \sqsubseteq R_{\delta}^{\prime}$.

We also need two intermediate lemmas. The first one is about the promotion rule for $\S$.

Lemma 2 (Promotion). Suppose that for any $\mathcal{S}$ such that $|R|_{\delta} \sqsubseteq \mathcal{S},(M, m) \in$ $|R \vdash A|_{\delta}^{\mathcal{S}}$ holds. Then for any $\mathcal{S}$ such that $|R|_{\delta+1} \sqsubseteq \mathcal{S}$, we have $(\S M, \S m+4) \in$ $|R \vdash \S A|_{\delta+1}^{\mathcal{S}}$.

Proof. Take $\mathcal{S}$ such that $|R|_{\delta+1} \sqsubseteq \mathcal{S},(E, e) \in\left(|R \vdash \S A|_{\delta+1}^{\mathcal{S}}\right)^{\perp \mathcal{S}}$ and $\left(S^{\prime}, s^{\prime}\right) \in \mathcal{S}$. We have $s^{\prime}=\S s_{\delta}+s_{0}$ with $\left(S^{\prime} \leq \delta, s_{\delta}\right) \in|R|_{\delta}$ (see Remark 6 ). We want to show that $\left(\left\langle! M, E, S^{\prime}\right\rangle, e\left(\S m+\mathbf{4}+s^{\prime}\right)\right) \in \mathbb{\Perp}$. By anti-reduction and $\leq$-saturation (by monoidality of $\S)$, it suffices to show $\left(\left\langle M, \S . E, S^{\prime}\right\rangle, e\left(\S\left(m+s_{\delta}\right)+s_{0}+\mathbf{3}\right)\right) \in \Perp$. We pose $\mathcal{S}^{\prime}=\left\{\left.(S, s)\left|\left(S, \S s+s_{0}\right) \in \mathcal{S} \wedge\left(S^{\leq \delta}, s\right) \in\right| R\right|_{\delta}\right\}$. Since $|R|_{\delta} \sqsubseteq \mathcal{S}^{\prime}$, and $\left(S^{\prime}, s_{\delta}\right) \in \mathcal{S}^{\prime}$, it is sufficient to prove $\left(\S . E, \lambda x . e\left(\S x+\mathbf{3}+s_{0}\right)\right) \in\left(|R \vdash A|_{\delta}^{\mathcal{S}^{\prime}}\right)^{\perp_{\mathcal{S}^{\prime}}}=$ $\|R \vdash A\|_{\delta}^{\perp \mathcal{S}^{\prime}}$. So let $(V, v) \in\|R \vdash A\|_{\delta}$ and $(S, s) \in \mathcal{S}^{\prime}$. We know that $(\S V, \S v) \in$ $\|R \vdash \S A\|_{\delta+1}$. Moreover, $\left(S, \S s+s_{0}\right) \in \mathcal{S}$. Since $(E, e) \in\|R \vdash \S A\|_{\delta+1}^{\perp \mathcal{S}}$, we have $\left(\langle\S V, E, S\rangle, e\left(\S v+\S s+s_{0}\right)\right) \in \Perp \Perp$. So by $\rightarrow$-saturation, $(\langle V, \S . E, S\rangle, e(\S v+\mathbf{1}+$ $\left.\left.\S s+s_{0}\right)\right) \in \Perp$. Hence, by distributivity of $\S$, we obtain $(\langle V, \S . e, S\rangle, e(\S(v+s)+$ $\left.\left.\mathbf{2}+\mathbf{1}+s_{0}\right)\right) \in \Perp$.

The proof of this last lemma is very important, since it justifies many design choices of our model.

- Its proof crucially relies on the fact that in the definition of the region context interpretation $|R|_{\delta}$, each value is guarded by a modality $\S$ and not by a modality !. Indeed, it requires the monoidality property, which is true for $\S$ but not for !: $\forall p, q \in \mathcal{M}, \S(p+q) \leq \S p+\S q$.

- It also relies on the fact that we can consider any set of store $\mathcal{S}$ such that $|R|_{\delta} \sqsubseteq \mathcal{S}$, which is also built-in in our interpretation of the linear arrow $\multimap$.

- It also justifies the fact that we need to consider $\mathcal{M}$-contexts, which are not only of the form $x \mapsto x+p$, since in this proof we use functions of the form $x \mapsto \S x+p$. 
We also prove a commutation lemma, which is needed when one wants to use biorthogonality in presence of call-by-value and modalities like! or $\S$.

Lemma 3 (Commutation). Suppose that $\forall(V, p) \in X,(M[V / x], q+p) \in Y$. Then for every $\mathcal{S}$, we have $\forall(V, p) \in X^{\perp_{\mathcal{S}} \perp_{\mathcal{S}}},(M[V / x], q+p+2) \in Y^{\perp_{\mathcal{S}} \perp_{\mathcal{S}}}$.

Proof. The proof mainly uses the property of $\rightarrow$-saturation and of $\rightarrow$-closure. Here are a series of implications.

$$
\begin{aligned}
& \forall(V, p) \in X,(M[V / x], q+p) \in Y \\
\Rightarrow & \forall(V, p) \in X, \forall(E, e) \in Y^{\perp_{\mathcal{S}}}, \forall(S, s) \in \mathcal{S},(\langle M[V / x], E, S\rangle, e(q+p+s)) \in \Perp \\
\Rightarrow & \forall(V, p) \in X, \forall(E, e) \in Y^{\perp_{\mathcal{S}}}, \forall(S, s) \in \mathcal{S}, \\
& (\langle V,(\lambda x \cdot M) \odot E, S\rangle, e(q+p+s+\mathbf{2})) \in \Perp \\
\Rightarrow & \forall(E, e) \in Y^{\perp_{\mathcal{S}}},((\lambda x . M) \odot E, \lambda x \cdot e(q+x+s+\mathbf{2})) \in X^{\perp_{\mathcal{S}}} \\
\Rightarrow & \forall(E, e) \in Y^{\perp_{\mathcal{S}}},((\lambda x . M) \odot E, \lambda x \cdot e(q+x+s+\mathbf{2})) \in X^{\perp_{\mathcal{S}} \perp_{\mathcal{S}} \perp_{\mathcal{S}}} \\
\Rightarrow & \forall(V, p) \in X^{\perp_{\mathcal{S}} \perp_{\mathcal{S}}}, \forall(E, e) \in Y^{\perp_{\mathcal{S}}}, \forall(S, s) \in \mathcal{S}, \\
& (\langle V,(\lambda x \cdot M) \odot E, S\rangle, e(q+p+s+\mathbf{2})) \in \Perp \\
\Rightarrow & \forall(V, p) \in X^{\perp_{\mathcal{S}} \perp_{\mathcal{S}}}, \forall(E, e) \in Y^{\perp_{\mathcal{S}}}, \forall(S, s) \in \mathcal{S}, \\
& (\langle M[V / x], E, S\rangle, e(q+p+s+\mathbf{2})) \in \Perp \\
\Rightarrow & \forall(V, p) \in X^{\perp_{\mathcal{S}} \perp_{\mathcal{S}}},(M[V / x], q+p+\mathbf{2}) \in Y^{\perp_{\mathcal{S}} \perp_{\mathcal{S}}}
\end{aligned}
$$

We can now prove Theorem 2

Proof. This theorem is proved by induction on the typing judgment. When we consider a value, we only prove the second statement, since it implies the first (by the properties of biorthogonality).

(v) This case is immediate by substitution.

(r), (u), (int) and (arith) These two cases are trivial, by definition of $\| R \vdash$ Unit $\|_{\delta}$ and $\left\|R \vdash \operatorname{Reg}_{r} A\right\|_{\delta}$.

(w) This case is just an application of $\leq$-saturation.

(fold), (unfold) The two fixpoint rules are easy, since we have $\|R \vdash \mu X . A\|_{\delta}=\| R \vdash$ $A[\mu X . A / X] \|_{\delta}$.

(lam) If the last rule used is the introduction of $\lambda$ :

$$
\frac{R ; \Gamma, y:(\lambda, A) \vdash^{\delta} N: B}{R ; \Gamma \vdash^{\delta} \lambda y \cdot N: A \multimap B}
$$

We take $(\bar{V}, \bar{p}) \Vdash^{\delta} \Gamma$. We denote by $N^{\prime}=N[\bar{V} / \bar{x}]$ and $p^{\prime}=\sum \bar{p}$. By induction hypothesis we know that for every $\mathcal{S}$ such that $|R|_{\delta} \sqsubseteq \mathcal{S}$ and every $\left(V^{\prime}, v^{\prime}\right) \in$ $\|R \vdash A\|_{\delta},\left(N^{\prime}\left[V^{\prime} / x\right], \llbracket N^{\prime} \rrbracket+p^{\prime}+v^{\prime}\right) \in|R \vdash B|_{\delta}^{\mathcal{S}}$. That means exactly that $\left(\lambda x . N^{\prime}, \llbracket \lambda x . N \rrbracket+p^{\prime}\right) \in\|R \vdash A \multimap B\|_{\delta}$ where $\llbracket \lambda x . N \rrbracket=\llbracket N \rrbracket$. 
(app) Here, to simplify the presentation, we suppose the contexts are empty (it does not change the argument).

$$
\frac{R ; \vdash^{\delta} M: A \multimap B \quad R ; \vdash^{\delta} N: A}{R ; \vdash^{\delta} M N: B}
$$

Take $\mathcal{S}$ such that $|R|_{\delta} \sqsubseteq \mathcal{S}$. By induction hypothesis we have

$$
\begin{aligned}
& (M, \llbracket M \rrbracket) \in|R \vdash A \multimap B|_{\delta}^{\mathcal{S}} \\
& (N, \llbracket N \rrbracket) \in|R \vdash A|_{\delta}^{\mathcal{S}}
\end{aligned}
$$

Take $(E, e) \in\left(|R \vdash B|_{\delta}^{\mathcal{S}}\right)^{\perp \mathcal{S}}$ and $(S, s) \in \mathcal{S}$. We want to show that

$$
(\langle M N, E, S\rangle, e(\llbracket M N \rrbracket+s)) \in \Perp
$$

where $\llbracket M N \rrbracket=\llbracket M \rrbracket+\llbracket N \rrbracket+3$. But $\langle M N, E, S\rangle \rightarrow\langle N, M \odot E, S\rangle$. Then, it suffices to show $(M \odot E, \lambda x . e(\llbracket M \rrbracket+\mathbf{2}+x)) \in\|R \vdash A\|_{\delta}{ }^{\perp_{\mathcal{S}}}$. Take $\left(V_{A}, v_{A}\right) \in$ $\|R \vdash A\|_{\delta}$ and $\left(S^{\prime}, s^{\prime}\right) \in \mathcal{S}$. Now we have to prove

$$
\left(V_{A}, M \odot E, S^{\prime}, e\left(\llbracket M \rrbracket+v_{A}+\mathbf{2}+s^{\prime}\right)\right) \in \Perp
$$

But $\left\langle V_{A}, M \odot E, S^{\prime}\right\rangle \rightarrow\left\langle M, V_{A} \cdot E, S^{\prime}\right\rangle$, so by $\rightarrow$-saturation we only have to prove $\left(V_{A} \cdot E, S^{\prime}, \lambda x . e\left(x+v_{A}+\mathbf{1}\right)\right) \in\|R \vdash A \multimap B\|_{\delta}^{\perp} \mathcal{S}$. Let $(\lambda x . P, p) \in \| R \vdash$ $A \multimap B \|_{\delta}$ and $(S, s) \in|R|_{\delta}$. We have $\left\langle\lambda x . P, V_{A} \cdot E, S\right\rangle \rightarrow\left\langle P\left[V_{A} / x\right], E, S\right\rangle$. But since $\left(P\left[V_{A} / x\right], p+v_{A}\right) \in|R \vdash B|_{\delta}^{\mathcal{S}}$, we have

$$
\left(\left\langle P\left[V_{A} / x\right], E, S\right\rangle, e\left(p+v_{A}+s\right)\right) \in \Perp
$$

Hence, we conclude that $\left(V_{A} \cdot E, \lambda x . e\left(x+v_{A}+\mathbf{1}\right)\right) \in\|R \vdash A \multimap B\|_{\delta}^{\perp \mathcal{S}}$ by $\rightarrow$-saturation.

(st) In this case, we have the following typing rule

$$
\begin{gathered}
r:(\delta, \S C) \in R \\
R ; \Gamma \vdash^{\delta-1} V: C \\
\hline R ; \Gamma \vdash^{\delta} \operatorname{set}(r, \S V): \text { Unit }
\end{gathered}
$$

Here, it is safe to consider that $\Gamma=\emptyset$ since $V$ is closed (the case $\Gamma \neq \emptyset$ is recovered from the case $\Gamma=\emptyset$ by $\leq$-saturation). Let $\mathcal{S}$ such that $|R|_{\delta} \sqsubseteq \mathcal{S}$, $(E, e) \in\|R \vdash \operatorname{Unit}\|_{\delta}^{\perp \mathcal{S}}$ and $(S, s) \in \mathcal{S}$. We want to prove

$$
(\langle\operatorname{set}(r, \S V), E, S\rangle, e(\S \llbracket V \rrbracket+\mathbf{1}+s)) \in \Perp
$$

But we have $(S, s+\S \llbracket V \rrbracket) \in \mathcal{S}$ so

$$
(\langle *, E, S \uplus\{r \rightarrow \S V\}\rangle, e(s+\S \llbracket V \rrbracket) \in \Perp
$$

Hence by $\rightarrow$-saturation and monotonicity of $e$, we obtain the result. 
(get) We now justify the get typing rule, which says that if $(r:(\delta, \S A) \in R$, then

$$
R ; \vdash^{\delta} \operatorname{get}(r): \S A
$$

Let $\mathcal{S}$ be such that $|R|_{\delta} \sqsubseteq \mathcal{S},(E, e) \in\|R \vdash \S A\|_{\delta}^{\perp \mathcal{S}}$ and $(S, s) \in \mathcal{S}$. So consider one possible decomposition $S=S^{\prime} \uplus\{r \rightarrow \S V\}$, then we can decompose $s=s^{\prime}+\S s_{V}$ with $\left(V, s_{V}\right) \in|R \vdash A|_{\delta-1}^{\mathcal{S}^{\prime}}$ for any $\mathcal{S}^{\prime}$ such that $|R|_{\delta-1} \sqsubseteq \mathcal{S}^{\prime}$. We then have

$$
\langle\operatorname{get}(r), E, S\rangle \rightarrow\left\langle\S V, E, S^{\prime}\right\rangle
$$

Moreover, by Lemma 2, $\left(\S V, \S s_{V}+\mathbf{4}\right) \in|R \vdash ! A|_{\delta}^{\mathcal{S}}$ and $\left(S^{\prime}, s^{\prime}\right) \in \mathcal{S}$. Hence,

$$
\left(\left\langle\S V, E, S^{\prime}\right\rangle, e\left(\S_{V}+\mathbf{4}+s^{\prime}\right)\right) \in \Perp
$$

Hence, by $\rightarrow$-saturation $(\langle\operatorname{get}(r), E, S\rangle, e(\mathbf{5}+s))$. We conclude that $(\operatorname{get}(r), \mathbf{5}) \in$ $|R \vdash \S A|_{\delta}^{\mathcal{S}}$.

(c) We want to justify the contraction rule

$$
\frac{R ; \Gamma, y:(!, A), z:(!, A) \vdash^{\delta} M: B}{R ; \Gamma, y:(!, A) \vdash^{\delta} M[y / z]: B}
$$

We take $(\bar{W}, \bar{p}) \Vdash^{\delta} \Gamma$. We denote by $M^{\prime}=M[\bar{W} / \bar{x}]$ and $p^{\prime}=\sum \bar{p}$. Let $\mathcal{S}$ such that $|R|_{\delta} \sqsubseteq \mathcal{S}$. We take $(V, v) \in\|R \vdash A\|_{\delta-1}$. By induction hypothesis we have $\left(M^{\prime}[V / y, V / z], \llbracket M \rrbracket+p^{\prime}+! v+! v\right) \in|R \vdash B|_{\delta}^{\mathcal{S}}$. Since $\|! p+! p\| \leq\|! p+\mathbf{2}\|$ for any $p \in \mathcal{M}$, we conclude that $\left(M^{\prime}[y / z][V / y], \llbracket M \rrbracket+p^{\prime}+! v\right) \in|R \vdash R|_{B}^{\delta} \mathcal{S}$. The case where $M^{\prime}$ is a value is similar.

$(!-$ prom $)$ The ! promotion rule is as follows.

$$
\frac{R ; x:(\lambda, A) \vdash^{\delta-1} V: B}{R ; x:(!, A) \vdash^{\delta} ! V: ! B}
$$

Let $\left(V^{\prime}, p\right) \in\|R \vdash A\|_{\delta-1}$. We know by induction that there is some $q$ such that $q \leq \llbracket V \rrbracket+p$ with $\left(V\left[V^{\prime} / x\right], q\right) \in\|R \vdash B\|_{\delta-1}$. We then have immediately that

$$
\left(! V\left[V^{\prime} / x\right], ! q\right) \in\|R \vdash ! B\|_{\delta}
$$

and so

$$
\left(! V\left[V^{\prime} / x\right], !(\llbracket V \rrbracket+p)\right) \in \overline{\|R \vdash ! B\|_{\delta}}
$$

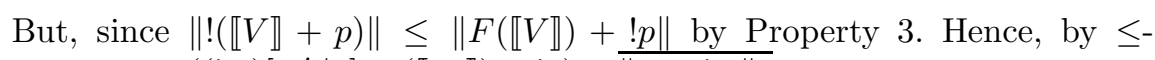
saturation, $\left((! V)\left[V^{\prime} / x\right], F(\llbracket M \rrbracket)+! p\right) \in \overline{\|R \vdash ! B\|_{\delta}}$.

(§- prom) Here, suppose the the rule is written

$$
\frac{R ; x_{1}:\left(\lambda, A_{1}\right), \ldots, x_{n}:\left(\lambda, A_{n}\right) \vdash^{\delta-1} M: C}{R ; x_{1}:\left(\S, A_{1}\right), \ldots, x_{n}:\left(\S, A_{n}\right) \vdash^{\delta} \S M: \S C}
$$

Let's take $\left(V_{i}, p_{i}\right) \in\left\|R \vdash A_{i}\right\|_{\delta-1}$ for $1 \leq i \leq n$ and $\left(W_{j}, q_{j}\right) \in\left\|R \vdash B_{j}\right\|_{\delta-1}$ for $1 \leq j \leq k$. We know by induction hypothesis that for any $\mathcal{S}$ such that 
XVIII

$|R|_{\delta-1} \sqsubseteq \mathcal{S}$, we have $\left(M\left[V_{i} / x_{i}, W_{j} / y_{j}\right], \llbracket M \rrbracket+\sum_{i} p_{i}+\sum_{j} q_{j}\right) \in|R \vdash C|_{\delta-1}^{\mathcal{S}}$.

Take $\mathcal{S}^{\prime}$ such that $|R|_{\delta} \sqsubseteq \mathcal{S}^{\prime}$. Hence, by Lemma 2 ,

$$
\left(\S M\left[V_{i} / x_{i}, W_{j} / y_{j}\right], \S\left(\llbracket M \rrbracket+\sum_{i} p_{i}+\sum_{j} q_{j}\right)+\mathbf{4}\right) \in|R \vdash \S C|_{\delta}^{\mathcal{S}^{\prime}}
$$

But, by Property 2 , we have $\left\|\S\left(\llbracket M \rrbracket+\sum_{i} p_{i}+\sum_{j} q_{j}\right)\right\| \leq \| \S \llbracket M \rrbracket+\sum_{i} \S p_{i}+$ $\sum_{j} ! q_{j} \|$. We can conclude

$$
\left(\S M\left[V_{i} / x_{i}, W_{j} / y_{j}\right], \llbracket \S M \rrbracket+\sum_{i} \S p_{i}+\sum_{j} ! q_{j}\right) \in|R \vdash \S C|_{\delta}^{\mathcal{S}^{\prime}}
$$

(§- elim) The rule is as follows (where the variables respectively associated to the contexts $\Gamma_{\lambda}, \Delta_{\lambda}, \Gamma_{!}, \Delta_{!}, \Gamma_{\S}$ and $\Delta_{\S}$ are noted $\left.\bar{x}, \overline{x^{\prime}}, \bar{y}, \overline{y^{\prime}}, \bar{z}, \overline{z^{\prime}}\right)$.

$$
\frac{R ; \Gamma \vdash^{\delta} V: \dagger B \quad R ; \Delta, x_{\S}:(\S, B) \vdash^{\delta} M: C}{R ; \Gamma, \Delta \vdash^{\delta} \text { let } \S x_{\S}=V \text { in } M: C}
$$

We take $\left(\overline{V_{\Gamma}}, \bar{p}\right) \Vdash^{\delta} \Gamma$ and $\left(\overline{V_{\Delta}}, \overline{p^{\prime}}\right) \Vdash^{\delta} \Delta$. We pose

$$
\begin{aligned}
V^{\prime} & =V\left[\overline{V_{\Gamma}} / \bar{x}\right] \\
p_{V} & =\sum \bar{p} \\
M^{\prime} & =M\left[\overline{V_{\Delta}} / \overline{x^{\prime}}\right] \\
p_{M} & =\sum \overline{p^{\prime}}
\end{aligned}
$$

Let's take $\mathcal{S}$ such that $|R|_{\delta} \sqsubseteq \mathcal{S}$. Then, by induction hypothesis, we know that if $\left(\S W, \S p_{W}\right) \in\|R \vdash \S B\|_{\delta}$,

$$
\left(M^{\prime}\left[W / x_{\S}\right], \llbracket M \rrbracket+p_{M}+\S p_{W}\right) \in|R \vdash C|_{\delta}^{\mathcal{S}}
$$

By $\rightarrow$-saturation (by considering a context), we obtain that for any $\left(\S W, \S p_{W}\right) \in$ $\|R \vdash \S B\|_{\delta}$

$$
\text { (let } \left.\S x_{\S}=\S W \text { in } M^{\prime}, \llbracket M \rrbracket+\mathbf{1}+p_{M}+\S p_{W}\right) \in|R \vdash C|_{\delta}^{\mathcal{S}}
$$

But we also know by induction hypothesis that $\left(V^{\prime}, \llbracket V \rrbracket+p_{V}\right) \in|R \vdash \S B|_{\delta}^{\mathcal{S}}$. Hence, by Lemma 3, since $|R \vdash C|_{\delta}^{\mathcal{S}}=\|R \vdash \S C\|_{\delta}{ }^{\perp} \perp_{\mathcal{S}}$, we obtain

$$
\text { (let } \left.\S x_{\S}=V^{\prime} \text { in } M^{\prime}, \llbracket M \rrbracket+\llbracket V \rrbracket+\mathbf{3}+p_{V}+p_{M}\right) \in|R \vdash C|_{\delta}^{\mathcal{S}}
$$

(! - elim) This case is completely similar to the previous case, except we have to replace every mention of $\S$ by !.

As a corollary of the adequacy theorem, we obtain the announced bounded-time termination theorem for $\lambda_{\mathrm{LAL}}^{\mathrm{Reg}, \mu}$ programs.

Proof (Termination theorem (Theorem 1)). This theorem is proved using adequacy together with Property 3. Indeed, we know that $\langle M, \diamond, \diamond\rangle$ terminates in at most $\|\llbracket M \rrbracket\|$ steps. But it is easy to see that only the promotion rules for $\S$ and ! make the value of $\|V a l M\|$ increases significantly: the degree of the third component of $\llbracket M \rrbracket$ (which is a polynomial) is bounded by a function of the depth of $M$. A similar argument is made more precise in [11, for instance. 


\section{Related Work}

Approximation modality - In a series of two papers, Nakano introduced a normalizing intuitionistic type system that features recursive types, which are guarded by a modality $\bullet$ (the approximation modality). Nakano also defines an indexed realizability semantics for this type system. The modality $\S$ plays in our work almost the same role as $\bullet$ : it makes the index increase. We claim that when we forget the quantitative part of our model, we obtain a model for a language with guarded references, that can be extended to handle control operators, based on a fragment of Nakano's type system: the only difference is that the $\bullet$ modality does not enjoy digging anymore (in presence of control operators, this principle would break normalization).

Stratified semantics for light logics - Several semantics for the "light" logics have been proposed, beginning with fibered phase models [16], a truthvalue semantics for LLL. We can also mention stratified coherent spaces [4]. These two models are indexed, like ours, but while the indexing is used to achieve completeness with respect to the logic, we use it to interpret fixpoints and references.

Reactive programming _ In [18, Krishnaswami \& al. have proposed a type system for a discrete-time reactive programming language that bounds the size of the data flow graph produced by programs. It is based on linear types and a Nakano-style approximation modality, thus bounding space consumption and allowing recursive definitions at the same time. They provide a denotational semantics based on both ultrametric semantics and length spaces. These latter, introduced by Hofmann [8] constitute the starting point of the quantitative realizability presented here.

\section{$5 \quad$ Research directions}

We see several possible directions we plan to explore.

Control operators - Since we use a biorthogonality-based model, it is natural to extend the language with control operators. Adding the call-cc operator can be done, but it requires to add a modality type ? for duplicable contexts. This involves some technical subtleties in the quantitative part, like the symmetrization of the notion of $\mathcal{M}$-contexts. Indeed, in our framework, a $\mathcal{M}$-context can be used to promote a weight associated to a term, but with this new ? type, a weight associated to a term would need to be able to promote a weight associated to a stack.

Multithreading — In the original work of Amadio and Madet [15, the language features regions but also multithreading. It is possible to add it to $\lambda_{\mathrm{LAL}}^{\mathrm{Reg}, \mu}$ but so far, it seems difficult to adapt the quantitative framework for this 
extension. It may be possible to adapt the notion of saturated store presented in [1, but with a boundedness requirement on it. We plan to explore this direction in the future.

\section{References}

1. R. Amadio. On stratified regions. Programming Languages and Systems, pages 210-225, 2009. 2, 2.2, 4, 5

2. A.W. Appel and D. McAllester. An indexed model of recursive types for foundational proof-carrying code. ACM Transactions on Programming Languages and Systems (TOPLAS), 23(5):657-683, 2001. 1

3. A. Asperti. Light affine logic. In Thirteenth Annual IEEE Symposium on Logic in Computer Science, 1998. Proceedings, pages 300-308, 1998. 1

4. P. Baillot. Stratified coherence spaces: a denotational semantics for light linear logic. Theoretical Computer Science, 318(1):29-55, 2004. 4

5. A. Brunel. Quantitative classical realizability. submitted, 2012. 10 1

6. U. Dal Lago, S. Martini, and D. Sangiorgi. Light logics and higher-order processes. Electronic Proceedings in Theoretical Computer Science, 41, 2010. 1

7. J-Y. Girard. Light linear logic. In Logic and Computational Complexity, pages 145-176. Springer, 1995. 1

8. M. Hofmann. Linear types and non-size-increasing polynomial time computation.

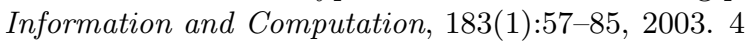

9. J-L. Krivine. Realizability in classical logic. Course notes of a series of lectures given in the University of Marseille, may 2004 (last revision: july 2005). Panoramas et syntheses, Société Mathématique de France, 2005. 1.

10. Y. Lafont. Soft linear logic and polynomial time. Theoretical Computer Science, 318(1-2):163-180, 2004. 1

11. U. Dal Lago and M. Hofmann. Bounded linear logic, revisited. In Pierre-Louis Curien, editor, Typed Lambda Calculi and Applications, volume 5608 of Lecture Notes in Computer Science, pages 80-94. 2009. 3.4

12. U. Dal Lago and M. Hofmann. A semantic proof of polytime soundness of light affine logic. Theory of Computing Systems, 46:673-689, 2010. 1] 3.1

13. U. Dal Lago and M. Hofmann. Realizability models and implicit complexity. Theoretical Computer Science, 412(20):2029 - 2047, 2011. Girard's Festschrift. 1

14. J. M. Lucassen and D. K. Gifford. Polymorphic effect systems. In Proceedings of the 15th ACM SIGPLAN-SIGACT symposium on Principles of programming languages, POPL '88, pages 47-57, New York, NY, USA, 1988. ACM. 2

15. A. Madet and R. Amadio. An elementary affine $\lambda$-calculus with multithreading and side effects. Typed Lambda Calculi and Applications, pages 138-152, 2011. 1 . 2.2, 5

16. M. Okada M.I. Kanovich and A. Scedrov. Phase semantics for light linear logic. Theoretical Computer Science, 294(3):525-549, 2003. 4

17. H. Nakano. A modality for recursion. In Logic in Computer Science, 2000. Proceedings. 15th Annual IEEE Symposium on, pages 255-266. IEEE, 2000. 1

18. N. Benton N.R Krishnaswami and J. Hoffmann. Higher-order functional reactive programming in bounded space. In Proceedings of the 39th annual ACM SIGPLAN-SIGACT symposium on Principles of programming languages, pages 45-58. ACM, 2012. 4 
19. M. Gaboardi P. Baillot and V. Mogbil. A polytime functional language from light linear logic. Programming Languages and Systems, pages 104-124, 2010. 1

20. Kazushige Terui. Light affine lambda calculus and polynomial time strong normalization. Archive for Mathematical Logic, 46(3-4):253-280, 2007. 2.2, 2.2 